\title{
Mejorar los Hábitos de Estudio de los Estudiantes que Repiten Curso en Primer Semestre de la Universidad
}

\author{
Improving Study Habits of Undergraduate Students that Failed \\ a First Semester Course
}

\author{
Ernesto Schiefelbein ${ }^{1 *}$
}

Alberto Lecaros ${ }^{2}$

${ }^{1}$ Universidad Autónoma de Chile. ${ }^{2}$ Universidad Metropolitana de Ciencias de la Educación

\begin{abstract}
Los estudiantes de pregrado que reprobaron una asignatura del primer semestre 2014, y tomaron un curso intensivo en Julio 2014, lograron mejores rendimientos académicos que un grupo que reprobó en el primer semestre 2013 y tomó el curso regular en el primer semestre 2014. El curso intensivo estimuló nuevos hábitos personales de estudio y sus profesores emplearon procesos de aprendizaje interactivo que se suelen usar en una "clase invertida" (flipped class). En este artículo se analizan los comentarios de los estudiantes sobre el tiempo dedicado a preparar su participación en las clases; las dudas que identificaron; la forma en que las resolvieron y otros aspectos positivos y negativos del curso intensivo. La mayoría de los estudiantes (sin diferencias de género) manifestó que habían mejorado sus hábitos de estudio, con respecto de la clase tradicional. Se realizará un seguimien to de los dos grupos en los próximos dos años para verificar si se mantiene el efecto positivo de los cambios de hábitos declarados por los estudiantes.
\end{abstract}

Descriptores: Innovación educacional, Estrategias educativas, Estudiante lento, Evaluación del estudiante, Deserción escolar.

\begin{abstract}
Undergraduate students who failed a course in the first semester 2014, and took an intensive course in July 2014, achieved a better academic performance than a group that failed a course in the first semester 2013 and repeated the regular course in the first semester 2014. The intensive course stimulated new study habits and their professors generated the interactive learning activities that are used in a "flipped class". This article analyzes comments made by students about the time spent preparing their participation in class, identifying doubts and eventually solving them. In addition, students commented on positive and negative aspects of the intensive course. Most students said the flipped class had improved their study habits with respect to the traditional classroom. The university will follow up the academic performance of both groups in the next two years to check whether the positive effect of changes in habits reported by students is maintained over time.
\end{abstract}

Keywords: Educational innovations, Educational strategies, Slow learners, Student evaluation, Dropping out. 


\section{Usar métodos efectivos de enseñanza para atender alumnos vulnerables}

Los profesores universitarios se seleccionan por su dominio del tema que deben enseñar, pero la mayoría no tiene una formación profesional en la manera de enseñar su tema. Por esto les cuesta enseñar de una manera diferente de la forma como ellos aprendieron. En este caso, el académico suele replicar la manera en que enseñaban los mejores profesores que tuvo en sus estudios universitarios. Esto explica que se continúe dictando charlas y los alumnos tomando notas (mientras escuchan) de lo que intuyen son los conceptos claves que presenta el profesor (HILT, 2012).

La mayor parte de los profesores no sabe que los conceptos que presenta en una charla de 20 minutos, los puede escribir en un texto de unas 1000 palabras. El estudiante podría leer ese texto (una vez) en menos de 5 minutos y, de paso, subrayar los conceptos principales (en vez de escribirlos en sus notas). Además puede volver a leer el texto las veces que quiera y buscar los sinónimos de las palabras desconocidas que le impedían entender el mensaje. El realizar esta preparación antes de la clase y el comenzar la clase aclarando las dudas, para luego profundizar los conceptos con ejercicios apropiados, son características esenciales de la "clase invertida" (flipped class). Este modelo de enseñanza se está aplicando con creciente éxito en diversos países para solucionar brechas en los conocimientos previos de los estudiantes.

No era necesario emplear mejores métodos de enseñanza cuando la universidad atendía una elite especialmente preparada para continuar los estudios de nivel superior. Pero la cobertura de la educación superior, en el grupo de chilenos de 18 a 24 años, creció de $16 \%$ en 1990 a 38\% en 2006 (Microdatos, 2008; Orellana, 2011). Este acelerado incremento ha permitido que la quinta parte de los estudiantes que ahora ingresan a la educación superior pertenezca a la mitad de la población con menor nivel socioeconómico. Una parte importante de este grupo de estudiantes ingresa a la universidad con menores conocimientos previos que los que ingresaban hace 20 ó 30 años atrás (Lecaros, 2016), y son poseedores de un menor capital social y cultural (Bernstein, 1973; Bourdieu y Passeron, 1979; Touraine, 2012).

La mayor (y creciente) heterogeneidad de los alumnos que ingresan a la universidad ha obligado a revisar la efectividad de los métodos de enseñanza, tanto en Chile como en otros países. "Solo $32 \%$ del total de estudiantes completa la educación media con una preparación académica suficiente para tener éxito en la universidad" (Greene y Forster, 2003, p. 1). Para compensar esa carencia, la "clase invertida" ha sido una estrategia exitosa. Los ensayos realizados en Chile han sido positivos, aunque ha sido difícil conseguir una implementación adecuada de ese método (McGinn y Schiefelbein, 2015; Schiefelbein y Zúñiga, 2002).

\section{Aplicación de la clase invertida en los cursos del $1^{\mathrm{er}}$ semestre 2012 al $4^{\circ}$ semestre 2013}

Durante el año 2011 se preparó la implementación de la clase invertida en cada uno de los 156 cursos que ofrecerían 700 profesores, en 30 carreras universitarias, a unos 5000 nuevos estudiantes que ingresarían al primer semestre en 2012 (Schiefelbein, 2012). 
La gran dispersión en los puntajes PSU, con que los alumnos ingresaron a la universidad, reflejó sus diferencias en el nivel de conocimientos previos. La baja competencia en lectura de un tercio de los alumnos representó un desafío especial para el proyecto.

La Dirección de Docencia de Pregrado, los Directores de Carrera y los docentes prepararon, entre septiembre 2011 y enero 2012, el programa detallado para cada clase y el material de lectura previa para cada una de las clases. Cada clase (del programa de un curso) describía los objetivos o temas de aprendizaje y tenía un "texto" en que se presentaba (en una o dos páginas), las definiciones y términos especializados que el profesor emplearía en esa clase. El texto también incluía aquellos conocimientos esenciales que los estudiantes debían dominar para entender los nuevos aprendizajes que se presentarían en "esa" clase (que suelen desconocer los ex-alumnos de algunos Liceos, en los que sólo se estudia una parte del currículo previsto para ese nivel).

En Julio de 2012, poco más de la mitad de los estudiantes encuestados creía que los profesores y administradores apoyaban la innovación y que los profesores estaban preparados para implementarla (McGinn y Schiefelbein, 2015). Estos porcentajes aumentaron considerablemente en los años siguientes (por la mayor proporción de docentes que formulaba preguntas al comenzar la clase y colocaba notas a las respuestas). Se observó una estrecha relación entre el número de elementos de la "clase invertida" que implementaba un profesor y la nota que asignaban los estudiantes a la innovación (esta información fue muy útil para mejorar la implementación en los años siguientes).

El desempeño de los estudiantes que leían regularmente el texto preparatorio mejoró con el tiempo, en relación con el desempeño de los estudiantes que no leían. Al final del tercer semestre, el promedio de notas de los que leían fue 4,82 en comparación con 4,63 para los que no leían. Era una diferencia estadísticamente significativa (sólo había 3 posibilidades en 100 que esa diferencia fuera resultado de la casualidad).

Estos positivos resultados llevaron a diseñar una implementación más cuidadosa en tres cursos de la Facultad de Ciencias de la Salud que tuvieron altos niveles de repetición: Anatomía General, Biología Celular y Química General. Se aprovechó las vacaciones de invierno de 2014 para ofrecer un curso intensivo que fuera supervisado de cerca por el equipo directivo.

El total de horas de clases del semestre regular se concentró en un curso de 12 días de duración con 6 horas cronológicas de clase cada día (3 horas en la mañana; 3 horas en la tarde y 2 horas de almuerzo e intermedios). Se revisaron los programas de cada clase y los textos de lectura previa. Los profesores revisaron las opiniones de los alumnos sobre los niveles de implementación (en 2012 y 2013) de los siete elementos esenciales del método de clase invertida. Finalmente, el equipo directivo asistió a las primeras clases de cada curso para comentar las desviaciones con respecto a la metodología acordada.

El grado de dificultad del examen final fue similar al del curso regular y el 95\% aprobó el curso (133 estudiantes aprobaron y 7 reprobaron). Este resultado se puede comparar con el $81 \%$ de aprobación de los 316 alumnos que reprobaron el curso en 2012 y lo repitieron en el primer semestre regular de 2013 (Lecaros, 2016).

Gracias a la cuidadosa preparación del curso intensivo se logró una correcta aplicación de la innovación en los tres cursos intensivos realizados en julio de 2014 y obtener un buen nivel de aprendizaje. Se aprovechó esta buena implementación para obtener 
opiniones de los estudiantes sobre algunas características de la manera en que se implementó y reunir información sobre los cambios de hábitos de los estudiantes. Esos antecedentes permitirán diseñar un estudio sobre el efecto de esos hábitos en los cursos que realicen en los próximos dos años.

\section{Encuesta aplicada a los estudiantes que completaron el curso intensivo}

La encuesta fue aplicada inmediatamente después del examen final de cada asignatura (que se cursaba por segunda vez), cuando los estudiantes desconocían su resultado académico en el curso. La encuesta no requería la identificación o nombre del alumno al momento de responderla, convirtiendo al instrumento en una recolección de comentarios de estudiantes anónimos, entendiendo por lo tanto que la encuesta se transforma en un instrumento de poder debido a que “...el comentario no tiene por cometido, cualesquiera que sean las técnicas utilizadas, más que el decir por fin lo que estaba articulado silenciosamente allá lejos" (Foucault, 1970, p. 15).

El Rector de la universidad invitó formalmente a responder la encuesta diciendo:

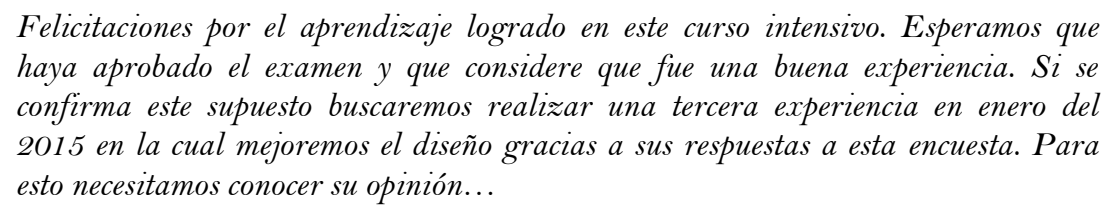

De esta manera, se solicitaba a los estudiantes responder con la verdad para mejorar la formación académica de sus pares.

Fue una encuesta de libre participación y tuvo un alto nivel de respuestas. De un total de 140 participantes, respondieron 106 estudiantes, lo cual representa una tasa de respuesta del $76 \%$. Tiene dieciséis preguntas, de las cuales siete son preguntas cerradas y factibles de categorizar en variables ordinales.

Las preguntas y respuestas de la encuesta se dividieron en dos categorías: cerradas y abiertas. Las siete preguntas cerradas se categorizaron como variables ordinales. Se constata inicialmente que no hay diferencias significativas según el género del estudiante. Esto permitió realizar el análisis para la muestra total (sin discriminar por género del estudiante).

El análisis de las preguntas cerradas se centrará en el "Tiempo (adicional a la clase) dedicado al estudio" y en el "Número de veces que leyó los materiales de estudio previo". El análisis de las preguntas abiertas se centrará en la "Percepción de aspectos positivos y negativos de la innovación basada en la clase invertida (flipped class). Esto permitirá sugerir, eventualmente, maneras de mejorar el impacto de la estrategia.

\section{Análisis de posibles diferencias en las respuestas según el género (sexo) del estudiante}

La variable "género" es nominal y tiene dos categorías (puede tomar dos valores). Para este tipo de variable es posible “... probar hipótesis considerando la distribución de casos entre las categorías, usando pruebas no paramétricas tales como la ji cuadrada o una 
prueba basada en la distribución binomial" (Siegel y Castellan, 1995, p. 45). Estas pruebas entregarían información respecto de diferencias en las respuestas del cuestionario según el sexo del estudiante (tabla 1).

Tabla 1. Prueba U de Mann-Whitney sobre diferencias estadísticas y género

\begin{tabular}{lcc}
\hline \multicolumn{1}{c}{ HIPóTESIS NULA } & SIG. & DECISIóN \\
\hline $\begin{array}{l}\text { Tiempo promedio que dedica a leer en los días de } \\
\text { autoestudio es el mismo entre las categorías de sexo }\end{array}$ & 0,944 & Retener la hipótesis nula \\
$\begin{array}{l}\text { Tiempo promedio que dedica a leer en los fines de semana } \\
\quad \text { es el mismo entre las categorías de sexo }\end{array}$ & 0,514 & Retener la hipótesis nula \\
$\begin{array}{l}\text { Número de veces que leyó el material en los días } \\
\text { destinados a autoestudio es el mismo entre las } \\
\quad \text { categorías de sexo }\end{array}$ & 0,513 & Retener la hipótesis nula \\
$\begin{array}{l}\text { Número de veces que leyó el material en los fines de } \\
\text { semana es el mismo entre las categorías de sexo }\end{array}$ & 0,443 & Retener la hipótesis nula \\
$\begin{array}{l}\text { La proporción que escribió las dudas que surgieron de la } \\
\text { lectura previa, es la misma entre las categorías sexo }\end{array}$ & 0,078 & Retener la hipótesis nula \\
$\begin{array}{l}\text { La proporción de dudas que fueron resueltas durante las } \\
\text { sesiones con los profesores, es la misma entre las } \\
\text { categorías sexo }\end{array}$ & 0,501 & Retener la hipótesis nula \\
\hline
\end{tabular}

Nota: El nivel de significancia es 0,05.

Fuente: Elaboración propia.

Los resultados del test de U Mann-Whitney sobre las hipótesis nulas que se presentan en la tabla 1 permiten afirmar, con un $95 \%$ de confianza, que no existen diferencias estadísticas significativas entre las respuestas y el sexo de los estudiantes.

\section{Tiempo promedio que dedican a leer en los días de autoestudio y en el fin de semana}

La mitad de los estudiantes declara leer entre dos y más horas, en cada uno de los cinco días dedicados al autoestudio (tabla 2) y son dos tercios los que declaran leer en el fin de semana. El mayor tiempo que los estudiantes dedicarían al estudio tendría un efecto positivo en el aprendizaje de los estudiantes (sin que la universidad tenga que dedicar recursos adicionales para lograrlo).

Tabla 2. Tiempo que dedican a leer en los días de autoestudio y en el fin de semana

\begin{tabular}{lcccc}
\hline TIEMPO PROMEDIO QUE & \multicolumn{2}{c}{ EN DÍAS DE AUTOESTUDIO } & \multicolumn{2}{c}{ EN EL FIN DE SEMANA } \\
\cline { 2 - 5 } \multicolumn{1}{c}{ DEDICó A LEER } & Frecuencia & Porcentaje & Frecuencia & Porcentaje \\
\hline No leí & 1 & 0,9 & 2 & 1,9 \\
Menos de 1 hora & 22 & 20,8 & 13 & 12,3 \\
1 Hora & 31 & 29,2 & 21 & 19,8 \\
2 Horas & 22 & 20,8 & 31 & 29,2 \\
3 Horas & 7 & 6,6 & 10 & 9,4 \\
Más de 3 horas & 23 & 21,7 & 29 & 27,4 \\
Total & 106 & 100,0 & 106 & 100,0 \\
\hline
\end{tabular}

Fuente: Elaboración propia.

Estos resultados sugieren que los cambios en los hábitos de lectura propuestos en la estrategia de aprendizaje de la clase invertida, están relacionados con las interrogaciones que a diario efectúan los profesores en el aula (a un alumno elegido al azar entre todos los participantes) y que califican con una nota que es considerada (con una baja ponderación) en la evaluación final de la asignatura. 


\section{Número de veces que el estudiante leyó el material e importancia de verificar que leyó}

Casi todos los alumnos habrían leído, al menos una vez, los materiales destinados a preparar una participación activa en la clase (tabla 3). El 84\% de los estudiantes declara que realizó al menos "dos lecturas completas" del material en los días de autoestudio y una cifra levemente menor (82\%) lo habría hecho en el fin de semana.

Esta alta proporción de alumnos que realizó dos lecturas completas sugiere que se generó una situación ideal para aclarar conceptos (y vocabulario) e identificar posibles dudas respecto a las materias leídas que, finalmente, se aclararían al comenzar la clase.

Tabla 3. Número de veces que leyó el material en los días destinado a autoestudio

\begin{tabular}{lcccc}
\hline \multicolumn{1}{c}{ NúMERO DE VECES QUE LEYÓ EL } & \multicolumn{2}{c}{ DÍAS DE AUTOESTUDIO } & \multicolumn{2}{c}{ FIN DE SEMANA } \\
\cline { 2 - 5 } MATERIAL & Frecuencia & Porcentaje & Frecuencia & Porcentaje \\
\hline Menos de una lectura completa & 4 & 3,8 & & \\
Una lectura completa & 13 & 12,3 & 19 & 17,9 \\
Dos lecturas completas & 50 & 47,2 & 50 & 47,2 \\
Más de dos lecturas completas & 39 & 36,8 & 37 & 34,9 \\
Total & 106 & 100,0 & 106 & 100,0 \\
\hline
\end{tabular}

Fuente: Elaboración propia.

Es probable que el buen nivel de lectura esté relacionado con el interés del estudiante para responder la pregunta efectuada por los profesores al inicio de cada clase. Un $94 \%$ de los estudiantes declaró que la nota de la interrogación inicial al azar realizada en cada clase, de las materias consideradas en las lecturas previas, "le ayudó en mejorar sus hábitos de estudio". Este dato es consistente con las investigaciones sobre cambios en los hábitos de los estudiantes (Dunlap, 2012; Leamnson, 1999; Nilson, 2010; Weimer, 2012).

\section{Identificar las dudas que surgen de la lectura previa y forma de resolverlas}

Un $85 \%$ de los estudiantes escribió las dudas que surgieron de la lectura previa (tabla 4). Este es un buen resultado pues el escribir las dudas, generadas al leer los textos, implica un esfuerzo por comprender la materia estudiada y también constituye una evidencia para el profesor de que el estudiante realizó efectivamente la lectura previa del texto.

Tabla 4. Grado en que se escriben y resuelven las dudas surgidas de la lectura previa

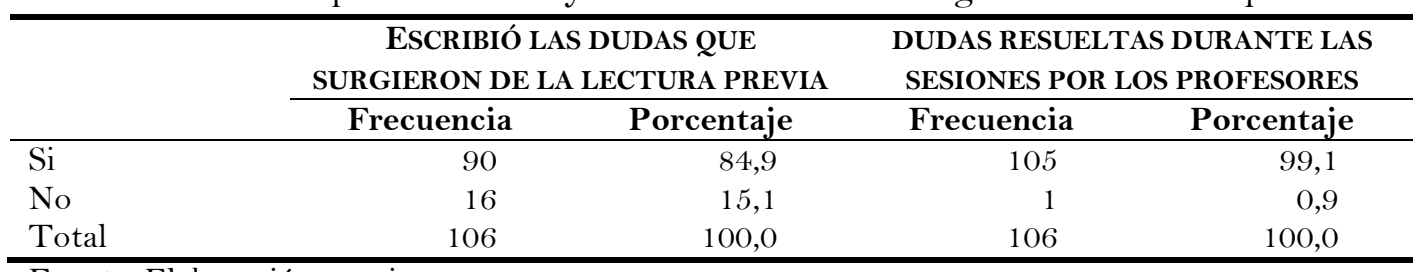

Fuente: Elaboración propia.

Un 99\% de los estudiantes declaró haber resuelto las dudas (generadas en las lecturas previas) durante las sesiones de clases con los profesores (tabla 4). Este alto porcentaje revela que se generó un espacio de interacción entre el profesor y las/los estudiantes que permitió aclarar las consultas en las clases o laboratorios y, también, retroalimentar al 
profesor respecto de las principales dificultades que enfrentan los estudiantes en cada tema. Este ejercicio no sólo es efectivo para el estudiante que consulta, sino que además favorece al grupo curso.

\section{Opiniones vertidas por los estudiantes sobre los cursos regulares e intensivos}

Primero se exponen las ideas más representativas de las respuestas de los estudiantes (sin intervenir ni la ortografía ni la redacción de las respuestas). Luego se muestra un cuadro denominado "nube de palabras", que es una representación visual de las palabras que conforman los textos escritos por los estudiantes. En ese cuadro, el tamaño de las palabras es proporcional a la frecuencia con que aparecen las palabras en el texto (se usó un software de libre disposición denominado "Tagul" que se obtiene en: https://tagul.com).

\subsection{Aspectos positivos del curso regular y del curso intensivo}

Entre los comentarios positivos más frecuentes sobre el curso Regular se mencionan los siguientes: "Mayor tiempo para prepararse para los exámenes", "Mayor tiempo para estudiar", "Más evaluaciones", “Apoyo visual”, "Presentaciones en diapositivas".

La nube de palabras que surge de las respuestas positivos de los estudiantes sobre el curso Regular parece destacar la duración del curso y las presentaciones en "power point" (tabla 5, columna izquierda). El principal aspecto positivo sería "disponer de más tiempo para estudiar la materia". Como segunda observación positiva se encuentra el apoyo visual empleado en las charlas tradicionales del profesor (esta observación se presenta como una debilidad en la aplicación de la clase intensiva).

Tabla 5. Aspectos positivos del curso regular y del curso intensivo

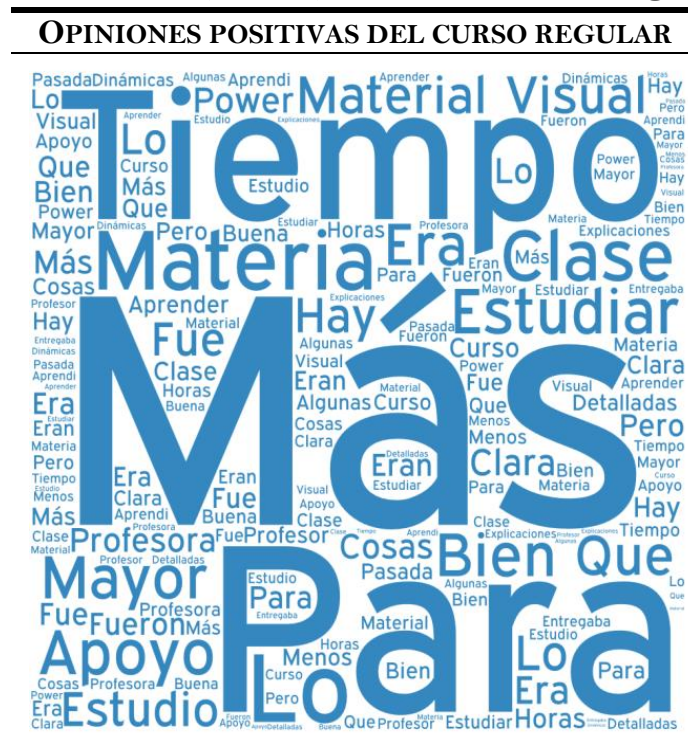

65 comentarios de 106 participantes

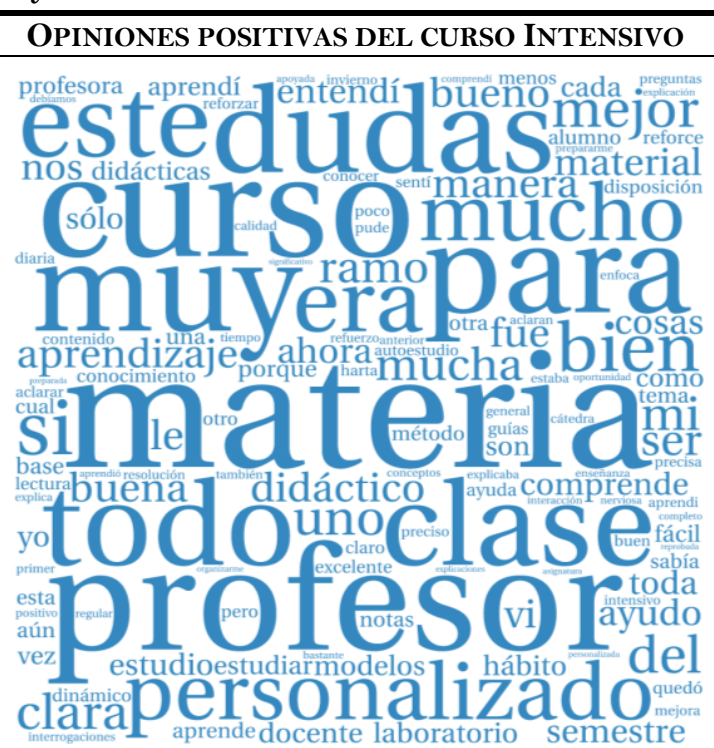

100 comentarios de 106 participantes

Fuente: Elaboración propia.

Entre los comentarios positivos más frecuentes de los estudiantes sobre el curso Intensivo (cuyos profesores emplean en mejor forma la clase invertida), se mencionan: 
"el método de enseñanza", "Los profesores tenían buena disposición para nuestro aprendizaje", "Interrogación diaria. Notas diarias", "Esta la materia más concisa, más clara y ahora en curso de invierno hubo por mi parte más interacción en cátedra y laboratorio." "Aprendí el porqué de las cosas".

Por su parte, la nube de palabras que surge de las respuestas también destaca el proceso de aprendizaje en la sala de clases (tabla 5, columna derecha). En general las apreciaciones de los aspectos positivos de la asignatura se refieren a la estrategia de aprendizaje usada en la clase invertida. Resalta la interacción con los profesores en las clases y sus respuestas a las dudas que surgen respecto de la materia tratada. También se destaca que el curso es personalizado, porque cada curso lo integran unos quince estudiantes a diferencia de las clases normales que son de unos treinta estudiantes. Perciben que el curso funciona mucho mejor lo que ayuda al aprendizaje. Finalmente los estudiantes mencionan un cambio en la didáctica del ramo o asignatura.

\subsection{Aspectos negativos del curso regular y del curso intensivo}

Entre los comentarios negativos más frecuentes sobre el curso Regular se mencionan los siguientes: "Poca profundización en lo básico y necesario para el curso. Muchos ejemplos irrelevantes. Ausencia de resolución de dudas", "La profesora no se daba el tiempo para explicar", "Demasiado material visual, a la hora de estudiar faltaba material", "Jamás pude comprender, ya que la metodología de la profesora no eran compatibles conmigo", "Creo que el profesor que nos tocó no enseño de la mejor manera", "Mi profesora explicaba muy mal y hablaba muy rápido y se le entendía poco”.

La nube de palabras que surge de los comentarios negativos de los estudiantes parece concentrarse en dos aspectos (tabla 6, columna izquierda). El primero se refiere al tipo de clase y a la presentación de la materia con medios visuales, pero con una muy baja interacción y escasa resolución de dudas en la clase. El segundo aspecto dice relación con las características de lo que consideran un mal profesor o profesora.

Tabla 6. Aspectos negativos del curso regular y del curso intensivo

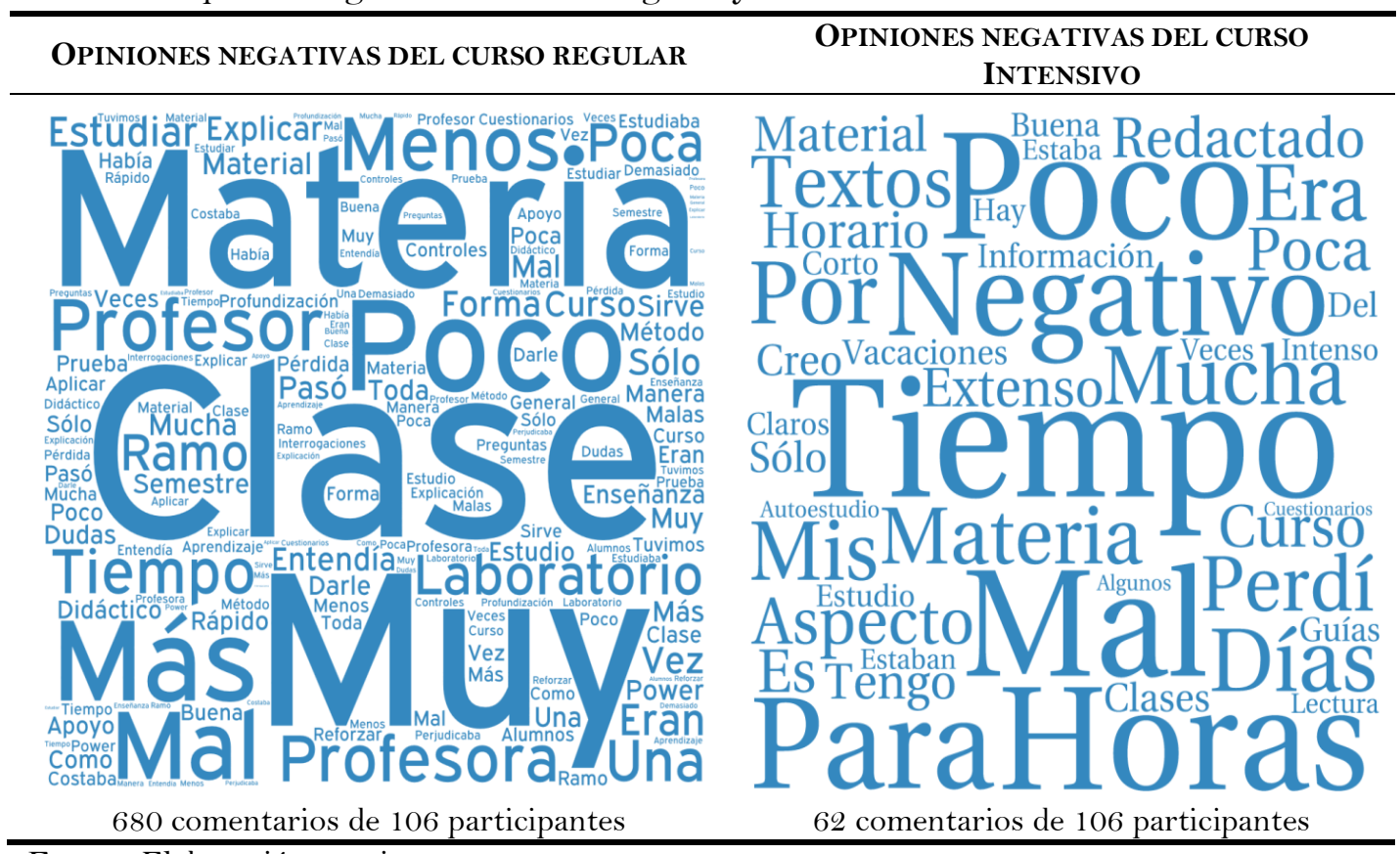

Fuente: Elaboración propia. 
Entre los comentarios negativos más frecuentes de los estudiantes sobre el curso Intensivo (que emplea la clase invertida), se mencionan los siguientes: "Poco tiempo en total. Jornada (diaria) demasiado extensa", "Lo negativo es que el material que entregaban estaba mal redactado o mal escrito y la letra a veces era muy pequeña", "Muy poco tiempo y mucha materia generalizada, no tan profundo como curso regular", "Mucha materia en pocas horas y días".

La nube de palabras que surge de los comentarios de carácter negativo de los estudiantes parece concentrarse en tres aspectos (tabla 6, columna derecha). Primero, los largos periodos de tiempo (expresado en horas) que deben pasar estudiando la materia. Luego mencionan que: perdí las vacaciones, ya que el curso intensivo fue dictado entre el periodo de vacaciones de invierno, entre el primer y segundo semestre. También consideran que hubo poco tiempo para preparar el examen final.

Entre los aspectos para mejorar la estrategia se menciona que había algunos textos que estaban mal redactados o mal diagramados lo que afectó la comprensión de la materia.

\section{Evaluación global de la experiencia de aprender con el método de clase invertida}

Se pidió a los estudiantes que comentaran si habrían aprobado el curso (en vez de fracasar en el primer semestre de 2013) si se hubiere usado el método de clase invertida (flipped class) y que explicaran la posible causa de haber obtenido un mejor resultado en esa oportunidad.

En general, los estudiantes respondieron que si hubiera usado la estrategia de aprendizaje basada en clase invertida la primera vez que cursó la asignatura de primer semestre hubiera aprobado el curso (tabla 7, columna izquierda).

Entre las principales razones para obtener un mejor aprendizaje se mencionan las siguientes: "Si, lo hubiese aprobado, ya que en las lecturas salían casos que yo no tenía en mi cuaderno debido a que muchas veces no alcanzaba a escribir algunos apuntes". "Por supuesto! El curso regular es sólo una breve pincelada que sólo entrega enunciados. La lectura previa orienta claramente y entrega un resumen de todo lo que se abordó en clase. Imprescindible la lectura previa", "Creo que si, puesto que en anatomía nunca nos evaluaron clase a clase y leyendo las lecturas previas sabíamos algo de los que nos iban a enseñar, por lo tanto no estábamos tan "colgados".

Finalmente, se pidió a cada estudiante "comparar la forma en que estudió el semestre anterior (cada semana tuvo clases de seis cátedras durante las 18 semanas del semestre), con un semestre en que se ofrecieran seis cursos intensivos consecutivos (cada tres semanas comienza uno nuevo), de modo que tienen el mismo número de horas de clases en ambas alternativas". Luego debían indicar cuál de las dos modalidades preferirían.

Entre las respuestas más representativas están las siguientes: "Curso intensivo: se me hizo más fácil entender la materia y estudiar", "Con respecto al curso ahora pude estudiar con más profundización, ya que sólo tenía que estudiar anatomía y no todos los ramos", "Yo creo que se aprendería mejor en el curso de invierno (intensivo), debido a que el tipo de estudio es más didáctico que el hecho en el semestre anterior", "Sería conveniente realizar plan piloto serio y medible para evaluar las desventajas (si las hubiese). Sin embargo creo que es más conveniente el curso intensivo", "En el semestre 
anterior estudie sólo de diapositivas de los profesores mientras que en este curso pude variar bibliografías, ya que, teníamos materiales anexos".

Tabla 7. Evaluación global del aprendizaje generado con la clase invertida

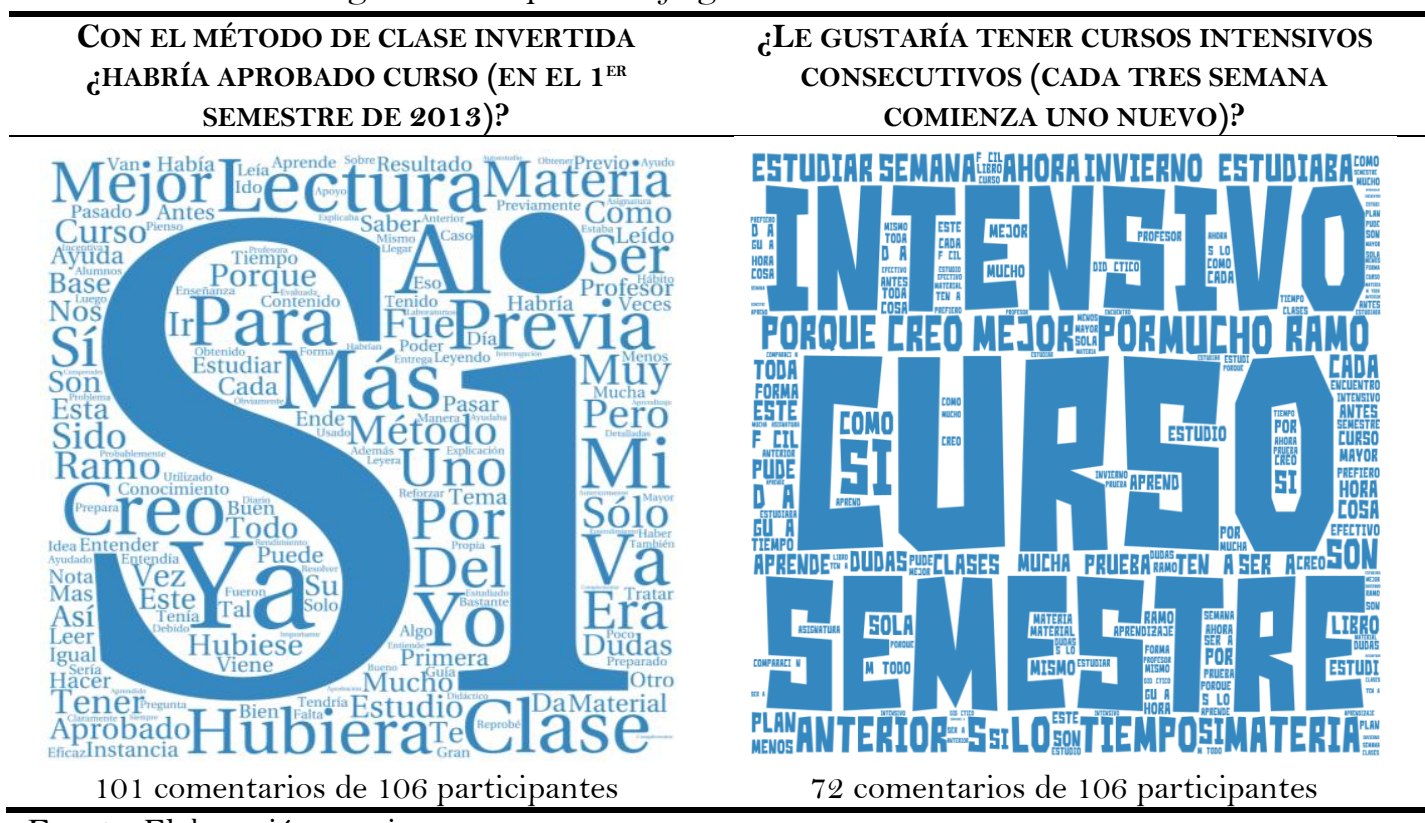

Fuente: Elaboración propia.

De la nube de palabras que surge de las respuestas de los estudiantes (tabla 7, columna derecha) parece que la mayoría de los estudiantes prefiere la estrategia basada en la clase invertida (flipped class) que muchos de ellos denominan curso intensivo.

\section{Conclusiones}

Los estudiantes repitientes que asistieron al curso intensivo declaran haber dedicado (en promedio) cerca de dos horas diarias (tabla 2) a leer el material seleccionado como lectura previa (en cada uno de los doce días que duró el curso). Este tiempo les permitió leer unas dos veces los materiales destinados a preparar su participación activa en cada clase (tabla 3).

La lectura en el curso intensivo parece ser bastante mayor que la que realizaban en los semestres regulares y esta actividad estaría estrechamente relacionada con el estar informado que la clase comenzaría con una interrogación con nota (a un alumno elegido al azar). Este incentivo habría influido en que un $85 \%$ de los estudiantes escribiera las dudas que surgieron de la lectura previa (tabla 4) y que se aclaraban al comenzar la siguiente clase.

Los estudiantes mencionan el proceso de aprendizaje personalizado en los cursos intensivos (tabla 5, columna derecha) y destacan la interacción con los profesores en las clases y sus respuestas a las dudas que surgen respecto de la materia tratada. Estos antecedentes explicarían que el grupo de alumnos repitentes en el 1er semestre 2014, que asistió al curso intensivo 2014, haya logrado una mejor tasa de aprobación (95\%) que el grupo de repitentes en el 1er semestre 2013 que asistió al curso regular en el 1er semestre 2014 (81\%). Los que aprobaron el curso intensivo no tuvieron vacación de 
invierno, pero evitaron extender en un año el tiempo necesario para graduarse en su carrera.

La correcta aplicación de la clase invertida en los tres cursos intensivos realizados en julio de 2014 (como resultado de una cuidadosa preparación de los profesores y materiales) permitió que los estudiantes obtuvieran buenos niveles de aprendizaje. Es por esto que los estudiantes creen que si se hubiera empleado esta estrategia de aprendizaje, en la primera vez que cursaron la asignatura de 1er semestre en 2014, habrían aprobado el curso (tabla 7, columna izquierda).

También se dispone de otras sugerencias de los estudiantes para continuar mejorando la implementación de la innovación en los próximos años. Además, se espera realizar un seguimiento de los estudiantes en su rendimiento académico, en los cursos que estudien durante los próximos dos años, para verificar si se mantiene el efecto positivo de los cambios experimentados en sus hábitos de estudio.

\section{Referencias}

Bernstein, B. (1973). Class, codes and control Applied studies towards a sociology of language. Londres: Routledge \& Kegan Paul.

Bourdieu, P. y Passeron, J. (1979). La distinction. París: de Minuit.

Dunlap, J. (2012). Encouraging students to read before class. Denver, CO: University of Colorado Denver.

Foucault, M. (1970). El orden del discurso. Buenos Aires: Tusquets Editores.

Greene, J. y Forster, M. (2003). Public high school graduation and college readiness rates in the United States. Nueva York, NY: Manhattan Institute for Policy Research.

HILT. (2012). The Harvard initiative on learning and teaching. Boston, MA: Harvard Magazine.

Leamnson, R. (1999). Thinking about teaching and learning: developing habits of learning with first year college and university students. Maryland, BA: University of Maryland.

Lecaros, A. (2016). Evaluación del uso de estrategia de aprendizaje basada en clase invertida en estudiantes universitarios de pregrado (Tesis de doctorado). Universidad Metropolitana de Ciencias de la Educación.

Microdatos. (2008). Estudio sobre causas de la deserción universitaria. Santiago: Universidad de Chile.

McGinn, N. y Schiefelbein, E. (2015). Getting students to read before class: Innovation in a university in Chile. Prospects, 45, 44-67. doi: 10.1007/s11125-015-9369-7

Nilson, L. B. (2010). Getting students to do the readings. En L. B. Nilson (Ed.), Teaching at its best: A research-based resource for college instructors (pp. 211-222). San Francisco, CA: JosseyBass

Orellana, V. (2011). Nuevos estudiantes y tendencias emergentes en la educación superior. En M. Jiménez y F. Lagos (Eds.), Nueva geografía de la educación superior y de los estudiantes (pp. 79-142). Santiago: Ediciones San Sebastián.

Schiefelbein, E. (2012). Chile: Improving access and quality to stop social unrest. International Higher Education, 68, 29-30.

Schiefelbein, E. y Zúñiga, R. (2002). El syllabus: Viviendo un aprendizaje autónomo. Santiago: Universidad Santo Tomás. 
Siegel, S. y Castellan N. J. (1995). Estadística no paramétrica. Ciudad de México: Trillas.

Touraine, A. (2012). ¿̇Podremos vivir juntos? Iguales y diferentes. Ciudad de México: Fondo de Cultura Económica.

Weimer, M. (2011). Two strategies for getting students to do the reading. The Teaching Professor, 25(1), 4-15.

\section{Breve CV de los autores}

\section{Ernesto Schiefelbein}

Enseña en Programas de Doctorado en Educación y es Investigador en Educación en la Universidad Autónoma de Chile (UA). Obtuvo su Ed. D. en Harvard University después de obtener en Chile sus títulos de Profesor e Ingeniero Comercial. Ha sido Director de la Oficina Regional de Educación de la UNESCO para América Latina y el Caribe y Educational Economist en el World Bank en Washington. Es autor o coautor de Learning to educate: proposals for the reconstruction in Developing cuntries (Unesco, 2017); "Projekt Erziehung. Ein Lehr-und Lernbuch (Ferdinand Schoningh, Germany, 2008); Universalization of Primary Education in Latin America (Institute of Developing Economics, Japan, 2007); Improve Teaching Methods or Perish, (Springer, Netherlands, 2005); Repensar la Educación, (Universidad Javeriana, Bogotá, 2005). ORCID ID: 0000oo01-7829-8669. Email: pschiefe@gmail.com

\section{Alberto Lecaros Alvarado}

Doctor en Educación de la Universidad Metropolitana de Ciencias de la Educación (2016). Magister en Educación de la Universidad de Chile (2010). Especialista en evaluación de proyectos por la Universidad de Chile (1995). Ingeniero en Administración Agroindustrial de la Universidad Tecnológica Metropolitana (1989). Enseña enseña el Taller de Ciencia y Tecnología como profesor titular en la Universidad Tecnológica Metropolitana de Chile. Investigador del Programa de Innovación en Ciencia y Arte PICALAB Universidad Metropolitana de Ciencias de la Educación. Administrador del Sistema de Monitoreo y Evaluación del Desempeño Profesional UMCE. ORCID ID: 0000-0002-1209-5700.Email: alberto.lecaros@umce.cl 